\title{
A study of the collapse speed of bubble clusters
}

\author{
Tezhuan Du ${ }^{\mathrm{a}, \mathrm{b}}$, Jingzhu Wang ${ }^{\mathrm{a}, \mathrm{b}}$, Yiwei Wang ${ }^{\mathrm{a}, \mathrm{b}}$, Chenguang Huang ${ }^{\mathrm{a}, \mathrm{b}, *}$ \\ a Institute of Mechanics, Chinese Academy of Sciences, Beijing, 100190, China \\ ${ }^{\mathrm{b}}$ School of Engineering Science, University of Chinese Academy of Sciences, Beijing 100049, China
}

\section{A R T I C L E I N F O}

Article history:

Received 24 May 2018

Revised 4 April 2020

Accepted 18 April 2020

Available online 26 April 2020

\section{Keywords:}

Bubble cluster

Collapse

Bubble distribution

Numerical simulation

\begin{abstract}
A B S T R A C T
The collapse process of bubble cluster is closely related to bubble-bubble interaction. Theoretical analysis and numerical simulation are adopted to study the collapse of bubble cluster with various distributions. The key parameters for bubble collapse, including bubble quantity, volume fraction, and dimensionless pressure, are acquired by dimensional analysis. The effects of key parameters on collapse of bubble cluster are investigated by direct numerical simulation. The numerical result shows that the collapsing speed of bubble cluster increases with the increase of bubble quantity and dimensionless pressure, decreases with the increase of volume fraction. A condensation rate is considered on the basis of bubble cluster with primitive cubic distributions. Square pyramid arrangement and random arrangement of bubbles are also simulated. A parameter study of the dimensionless bubble distance bubble cluster with random arrangement shows that a larger distance generally results in a larger collapse speed of bubble cluster.
\end{abstract}

(c) 2020 Elsevier Ltd. All rights reserved.

\section{Introduction}

Cavitation is a key issue in high-speed underwater propulsion and can result in structure failure (Bark et al., 2011) and acoustic emissions (Kim et al., 2014). Cloud cavitation, which consists of a large number of small bubbles, is a common type of cavitation for high-speed vessels and projectiles. Pressure pulse generated by bubble collapse in cloud cavitation is a major cause of structural failure and noise radiation.

Due to the geometric complexity of a collapsing bubble cluster and the asymmetric behavior of each bubble, simplified homogeneous models were first developed to study the characteristic of bubbly mixture flow (van Wijngaarden, 1964; Kedrinskii and Mørch, 1725; Omta, 1987; D'Agostino and Brennen, 1989). With the rapid development of computer capability and simulating technology, considerable efforts were devoted in the last decades to numerical simulations of cavitating flow. Most of these studies focused on homogeneous flow modeling, which comprises the Navier-Stokes $(\mathrm{N}-\mathrm{S})$ equations of the mixture phase (Coutier-Delgosha et al., 2007; Merkle et al., 2001; Kunz et al., 2000; Kubota and Kato, 1989; Singhal et al., 2002; Schnerr and Sauer, 2001; Zwart et al., 2004). The phase change between liquid and vapor is usually modeled by the transport equation of the vapor phase with phase change rate as source term. Merkle (Merkle et al., 2001), Kunz (Kunz et al., 2000), Kubota

\footnotetext{
* Corresponding author.

E-mail address: huangcg@imech.ac.cn (C. Huang).
}

(Kubota and Kato, 1989), Singhal (Singhal et al., 2002), Schnerr (Schnerr and Sauer, 2001), and Zwart (Zwart et al., 2004) proposed several cavitation models based on phase-change method; these models are utilized to simulate cavitating flows in many applications (Owis F and Nayfeh, 2004; Ji et al., 2012, Yakubov et al., 2015; Huang et al., 2013; Huang et al., 2014; Morgut et al., 2011; Huang et al., 2012). Ji et al. (Ji et al., 2012) used Zwart model to simulate cavitating flows around a conventional marine propeller in a non-uniform wake and predict the excited pressure fluctuations. Huang et al. investigated cavitating flows around a pitching hydrofoil and vortex-cavitation interactions using Kubato model (Huang et al., 2013; Huang et al., 2014). To include the bubble-bubble interactions, Maiga et al. (Maiga et al., 2018) proposed a new cavitation model based on the mutual interaction between two spherical bubbles of different sizes. Another approach to simulate the cavitating flow is Eulerian-Lagrangian method (Chahine et al., 2014; Ma et al., May 2015; Ma et al., January 2018), modeling each bubble base on Rayleigh-Plesset equation.

Bubble-bubble interactions are usually neglected in cavitation models based on the following hypothesis. Bubbles grow or collapse synchronously and remain spherical during this process. Nevertheless, bubble clusters with different distributions may lead to various collapse pressures due to the interaction of bubbles under identical vapor volume fractions. Therefore, a numerical model that considers bubble-bubble interaction is essential to improving the accuracy of predicting bubble collapse pressure. Achieving theoretical analysis of a collapsing bubble cluster is difficult due to the complex asymmetric behavior of each bubble. Optical diagnostics within the bubble cluster are also challenged by the large index of 
refraction mismatch at the bubble surfaces. Therefore, direct numerical simulation is an important approach in the investigation of bubble cluster dynamics. Zhang and Yao (Zhang et al., 2008) employed boundary element method to study the factors that affect the expansion, collapse, and movement of multiple bubbles. Zhang and Shao (Zhang et al., 2013) examined the influences of bubble interaction on bubble cluster by direct numerical simulation and showed that the collapse of central bubble in a multibubble system contains delay and acceleration stages. Chahine and Duraiswami (Chahine G and Duraiswami, 1992) simulated multiple bubbles by boundary integral method and revealed the presence of large non-spherical bubble deformations and inward jets upon collapse. Bremond et al. provided a similar picture of collapse with large aspherical bubble deformations and re-entrant jetting (Bremond et al., 2006). Tiwari et al. (Tiwari et al., 2015) simulated the expansion and subsequent collapse of a hemispherical cluster of 50 bubbles adjacent to a plane rigid wall, wherein geometricfocused bubbles generate high impulsive pressures. Maeda and Colonius (Maeda and Colonius, 2018) present a coupled EulerianLagrangian method to simulate cloud cavitation in a compressible liquid and capture the bubble-scattered acoustics. Hsiao et al. (Hsiao et al., May 2018) investigate the influence of driving frequency of sinusoidal pressure field on the collapse of bubble cloud dynamics near a rigid wall.

However, the influence of bubble distributions on collapse requires further study to introduce bubble interactions into the cavitation model. In the present study, we focus on bubble-bubble interaction in the collapse stage of bubble cloud. Non-dimensional parameters for collapse of bubble cluster are acquired by dimensional analysis. Primitive cubic lattice distribution is selected to examine the influence of these parameters. An empirical formula for collapse speed of bubble cluster is achieved by direct numerical simulation. A condensation rate based on bubble cluster is promoted. Furthermore, collapse of bubble clusters with various distributions is simulated to discuss the representativeness of primitive cubic lattice arrangement and the applicability of the present empirical formula.

\section{Dimensional analysis of bubble cluster collapse}

We consider a bubble cluster collapse under pressure $p_{\infty}$ (Fig. 1). Assuming that all bubbles are spherical and in the equilibrium state at the beginning.

The related parameters and dimensions are listed as follows:

Time: $t[T]$

Characteristic length of bubble cluster: $a[L]$

Initial bubble radii: $R_{0}[L]$

Population of bubbles per unit volume: $n\left[\frac{1}{L^{3}}\right]$

Material parameters: liquid density $\rho_{L}\left[\frac{M}{L^{3}}\right]$, liquid viscosity $\mu_{L}\left[\frac{M}{L T}\right]$, surface tension $S\left[\frac{M}{T^{2}}\right]$, reference density of gas $\rho_{A 0}\left[\frac{M}{L^{3}}\right]$, gas viscosity $\mu_{A}\left[\frac{M}{L T}\right]$, ratio of specific heats of gas $\gamma[1]$

Surrounding pressure: $p_{\infty}\left[\frac{M}{L T^{2}}\right]$

Pressure inside bubbles: $p_{B}\left[\frac{M}{L T^{2}}\right]$

The average variation rate of volume of all bubbles can be expressed in the following relationship:

$\overline{\dot{V}}=f\left(R_{0}, a ; n ; \rho_{L}, \mu_{L}, S, \rho_{A 0}, \mu_{A}, \gamma ; p_{\infty}, p_{B}\right)$

We take $R_{0}, \rho_{L}$, and $p_{\infty}-p_{B}$ as a unit system produces dimensionless form:

$\frac{\overline{\dot{V}}}{\frac{4}{3} \pi R_{0}^{3} / T_{C}}=f\left(\alpha ; N ; \frac{\mu_{L}}{\rho_{L} R_{0}^{2} / T_{C}}, \frac{S}{\rho_{L} R_{0}^{3} / T_{C}^{2}}, \frac{\rho_{A 0}}{\rho_{L}}, \frac{\mu_{A}}{\rho_{L} R_{0}^{2} / T_{C}}, \gamma, \frac{p_{\infty}-p_{B}}{p_{\infty}}\right)$

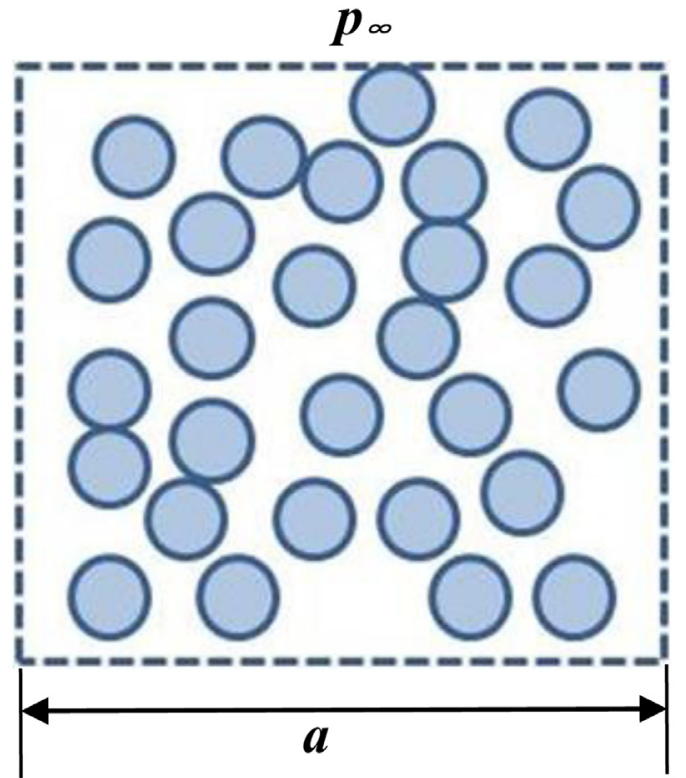

Fig. 1. Schematic of bubble cluster.

where $T_{C}=0.915 R_{0} \sqrt{\frac{\rho_{L}}{p_{\infty}-p_{B}}}$ is the collapse time of single bubble, $\alpha$ is the volume fraction of bubbles, and $N$ is the total bubble number. The collapse of bubbles is controlled by pressure difference and inertial force, Therefore, viscosity and surface tension can be neglected. For a given material, $\frac{\rho_{A 0}}{\rho_{L}}$ and $\gamma$ are constant. Thus, the equation can be changed to:

$\frac{\overline{\dot{V}}}{\frac{4}{3} \pi R_{0}^{3} / T_{C}}=f\left(\alpha ; N ; p^{\prime}\right)$

where $p^{\prime}=\frac{p_{\infty}-p_{B}}{p_{\infty}}$ represents the non-dimensional driving pressure. $\dot{V}_{0}=\frac{\frac{4}{3} \pi R_{0}^{3}}{T_{C}}$ represents the volume variation rate of single isolated bubble. Thus, $\overline{\dot{V}}=\frac{\overline{\dot{V}}}{\dot{V}_{0}}$ is the non-dimensional volume variation rate of bubble cluster. Eq. (3) shows the following three key parameters: $\alpha, N$, and $p^{\prime}$. To provide the specific relation, we can assume the following:

$\overline{\dot{V}}^{\prime}=c \alpha^{k_{1}} N^{k_{2}} p^{\prime k_{3}}$.

Empirical parameters $c, k_{1}, k_{2}, k_{3}$ can be determined by direct simulation of bubble cluster collapse, respectively. The nondimensional volume variation rate is used to denote the collapse of bubble cluster.

\section{Direct simulations of bubble cluster collapse}

\subsection{Governing equations}

The basic approach consists of unsteady Navier-Stokes equations in the mixture phase and the continuity equation in the vapor phase. The VOF method can model two or more immiscible fluids by solving a single set of momentum equations and tracking the volume fraction of each of the fluids throughout the domain. The Navier-Stokes equations of compressible flow are listed as follows:

$$
\frac{\partial \rho}{\partial t}+\nabla \cdot(\rho \mathbf{v})=0
$$

$\frac{\partial(\rho \mathbf{v})}{\partial t}+\nabla \cdot(\rho \mathbf{v v})=-\nabla p+\nabla \cdot(2 \mu \mathbf{S})-\frac{2}{3} \nabla(\mu \nabla \cdot \mathbf{v})$, 
$\frac{\partial(\rho E)}{\partial t}+\nabla \cdot[\mathbf{v}(\rho E+p)]=\nabla \cdot\left[k_{e f f} \nabla T+\left(2 \mu \mathbf{S}-\frac{2}{3} \nabla \cdot \mathbf{v I}\right) \cdot \mathbf{v}\right]$

where $\mathbf{S}=\frac{1}{2}\left(\nabla \mathbf{v}+\nabla \mathbf{v}^{T}\right)$ stands for the strain rate tensor. The density and viscosity can be expressed by volume fraction of the vapor phase $\alpha_{v}$ (Bensow and Bark, 2010; Bensow and Bark, 2010; Wang and Ostoja-Starzewski, 2007):

$\rho=\alpha_{v} \rho_{v}+\left(1-\alpha_{v}\right) \rho_{l}$,

$\mu=\alpha_{v} \mu_{v}+\left(1-\alpha_{v}\right) \mu_{l}$

Subscripts $l$ and $v$ represent the water and vapor, respectively.

Turbulent flow is simulated by large eddy simulation (LES), which is based on an assumption that the large- and small-scale eddies are computed by the unsteady Navier-Stokes equations and the subgrid stress model, respectively. In the method of the LES, $\varphi$ is decomposed into large-scale quantity $\bar{\varphi}$ and small-scale quantity $\varphi^{\prime} . \bar{\varphi}$ can be expressed as follows:

$\bar{\varphi}(\mathbf{x})=\int_{D} \varphi\left(\mathbf{x}^{\prime}\right) G\left(\mathbf{x}, \mathbf{x}^{\prime}\right) d \mathbf{x}^{\prime}$,

where $G\left(\mathbf{x}, \mathbf{x}^{\prime}\right)$ is the filter function, which can be expressed as:

$G\left(\mathbf{x}, \mathbf{x}^{\prime}\right)=\left\{\begin{array}{l}1 / V \mathbf{x}^{\prime} \in \Delta \\ 0 \mathbf{x}^{\prime} \notin \Delta\end{array}\right.$,

where $V$ is the volume of a computational cell $\Delta$.

Introducing the following density-weighted (or Favre) filtering operator is convenient for compressible flows:

$\tilde{\varphi}=\frac{\overline{\rho \varphi}}{\bar{\rho}}$. form:

The Favre-filtered Navier-Stokes equation takes the following

$\frac{\partial \bar{\rho}}{\partial t}+\nabla \cdot(\bar{\rho} \tilde{\mathbf{v}})=0$

$\frac{\partial(\bar{\rho} \tilde{\mathbf{v}})}{\partial t}+\nabla \cdot(\bar{\rho} \tilde{\mathbf{v}})=-\nabla \bar{p}+\nabla \cdot(2 \mu \overline{\mathbf{S}})-\frac{2}{3} \nabla(\mu \nabla \cdot \overline{\mathbf{v}})+\boldsymbol{\tau}$.

The compressible form of the subgrid stress tensor is defined as:

$\boldsymbol{\tau}=\bar{\rho} \widetilde{\mathbf{v}}-\bar{\rho} \tilde{\mathbf{v}} \tilde{\mathbf{v}}$.

The subgrid-scale turbulence model in this study employs the Boussinesq hypothesis by computing subgrid stresses from the following:

$\tau_{i j}-\frac{1}{3} \tau_{k k} \delta_{i j}=-2 \mu_{t} \bar{S}_{i j}$

The subgrid-scale turbulent viscosity. $\mu_{t}$ is modeled by Smagorinsky-Lilly model in the present study and the value of Smagorinsky constant $C_{S}$ is set to be 0.1 . The isotropic part of the subgrid-scale stresses that $\frac{1}{3} \tau_{k k} \delta_{i j}$ is not modeled but added to the filtered static pressure term. $\bar{S}_{i j}$ is the strain rate tensor for the resolved scale, which is defined by

$\overline{\mathbf{S}}=\frac{1}{2}\left(\nabla \overline{\mathbf{v}}+\nabla \overline{\mathbf{v}}^{T}\right)$.

The direct simulation of bubble cluster collapse is carried out in the commercial software of FLUENT. The equations are solved using SIMPLE algorithm based on finite volume method (FVM) (ANSYS Fluent12 2008; Xu et al., 2018). For the spatial discretization, the PRESTO! scheme, bounded central differencing scheme and first-order upwind scheme are used for spatial interpolation of pressure, momentum and energy respectively. The first-order implicit scheme is used for time discretization. For the VOF method, the geometric reconstruction scheme is adopted to represent the interface between fluids using a piecewise-linear approach.

\subsection{Computational model}

Consider a cubic bubble cluster with primitive cubic lattice distribution, wherein bubbles are arranged in $i \times i \times i$ order. A cubic computational domain of $(375 \times 375 \times 375) \mathrm{mm}^{3}$ is created with boundary conditions of pressure inlet. All the bubbles lie in a smaller cube of $(62.5 \times 62.5 \times 62.5) \mathrm{mm}^{3}$ as shown in Fig. 2 . The bubble is discretized with a mesh of minimum spacing $\Delta x_{\text {min }}$ such that $\Delta x_{\min } \leq R_{0} / 16$. Here $R_{0}$ is the initial bubble radius. The initial pressure inside the computational domain is set as $p_{B}$, and the pressure at the outer boundary is $p_{\infty}$.

A single bubble collapse has been simulated using different mesh scales. In this case, the pressure at far field and the initial pressure in bubble are set to be $p_{\infty}=101,325 \mathrm{~Pa}$ and $p_{B}=20,000 \mathrm{~Pa}$ respectively, and the initial bubble radius is $R_{0}=5 \mathrm{~mm}$. The bubble is discretized with a mesh of minimum spacing $\Delta \mathrm{x}_{\min }=R_{0} / 16, R_{0} / 32, R_{0} / 64$. where $R_{0}$ is the initial bubble radius. The simulation results are compared to the Rayleigh Plesset equation (Fig. 3). Fig. 3 shows that simulations can predict a reasonable the volume history of an isolated bubble except for the last stage of collapse $(t=0.5-0.55 \mathrm{~ms})$. The relative error of averaged collapse speed between numerical simulation and Rayleigh Plesset equation is less than $5 \%(3.2 \%, 3.2 \%, 4.4 \%)$. Note that if the focus of the cluster simulation is to accurately capture the bubble surface and the associated jet dynamics during collapse, then a finer mesh is needed for grid convergence. Such a study is done by Tiwari et al. (Tiwari et al., 2013), in which, a new, simple, and computationally efficient interface capturing scheme is used. However, since the focus of the present work is on the time variance

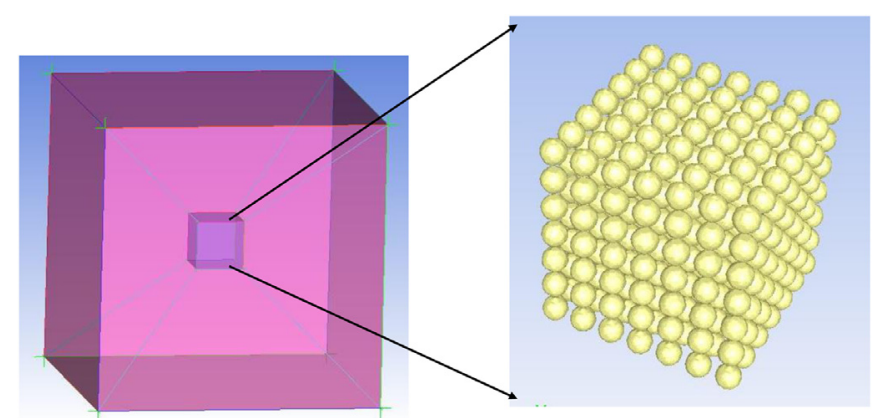

Fig. 2. Flow field and numerical model

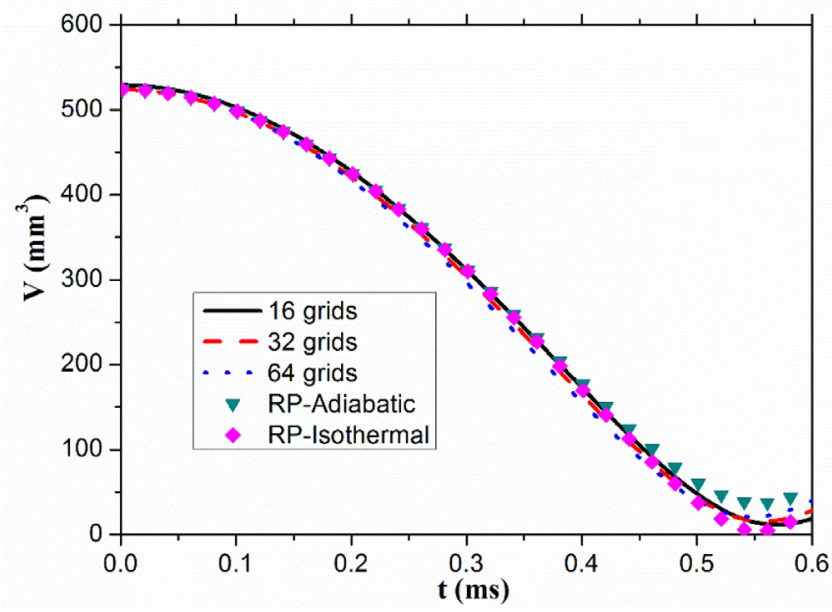

Fig. 3. Comparisons of CFD simulation and Rayleigh Plesset equation on time variation of the bubble volume for $R_{0}=5 \mathrm{~mm}$. 


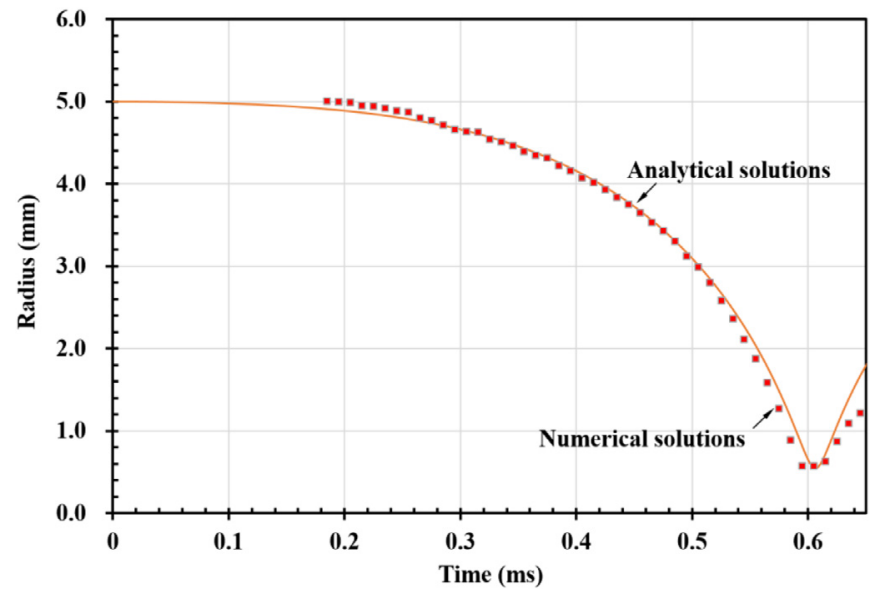

Fig. 4. Comparison of radial dynamic of $5 \mathrm{~mm}$ radius center bubble between analytical solution model and Numerical solution.

of bubble volume, the minimum spacing $\Delta x_{\min }$ is required to be $\Delta x_{\min } \leq R_{0} / 16$.

To verify the accuracy of the numerical method used in the present study, we need to compare the numerical solution with the analytical solution of the motion of the center bubble. According to the model proposed by Doinikov (Doinikov, 2004); Bremond et al. (Bremond et al., 2006), the governed equations for bubbles are:

$R_{i} \ddot{R}_{i}+\frac{3}{2} \dot{R}_{i}^{2}=\frac{p\left(R_{i}\right)-p_{l}(t)}{\rho}-\sum_{j \neq i} \frac{R_{j}^{2} \ddot{R}_{j}+2 R_{j} \dot{R}_{j}^{2}}{r_{i j}}$

where $p\left(R_{\mathrm{i}}\right)$ is the pressure on the bubble wall, $p_{\mathrm{l}}$ is the pressure of the liquid at infinity, and $r_{\mathrm{ij}}$ is the distance between a bubble $i$ and another bubble $j$.

Therefore, the equation of the center bubble is expressed in Eq. (18).

$R_{i} \ddot{R}_{i}+\frac{3}{2} \dot{R}_{i}^{2}=\frac{p\left(R_{i}\right)-p_{l}(t)}{\rho}-s\left(R_{j}^{2} \ddot{R}_{j}+2 R_{j} \dot{R}_{j}^{2}\right)$

The last term of this equation represents the effect of the bubble-bubble interaction. Based on the study by Yasui et al. (Yasui and Kato, 2012), $s$ is the coupling strength of a bubble cloud and is expressed as follows:

$s=\sum_{j=1}^{n} \frac{1}{r_{i j}}=\int_{l_{\min }}^{l_{\max }} \frac{4 \pi r^{2} n}{r} d r=2 \pi n\left(l_{\max }^{2}-l_{\min }^{2}\right)$

where the summation is for all the bubbles, $n$ is the number density of the bubbles, $l_{\max }$ is the radius of the bubble cloud, and $l_{\min }$ is the distance between the center bubble and the nearest bubble.

The fourth-order Runge-Kutta method is used to solve the bubble equation. The analytical solution is shown in Fig. 4. In the analysis, the initial radius and internal pressure of the center bubble are $5 \mathrm{~mm}$ and $2 \times 10^{4} \mathrm{~Pa}$, respectively. From the figure, it is found that the center bubble contracts to the minimum size at about $t=0.605 \mathrm{~ms}$ when the surrounding pressure is an atmosphere. For the contraction of the bubble, the numerical solution used in the manuscript shows good agreements with the analytical solutions. In the present study, we focus on the bubble dynamic during the first collapse. Consequently, the numerical method is thought to be reasonable.

In the bubble cluster, collapsing shock waves induced by a bubble can interact with other bubbles around it. Therefore, it is necessary to investigate the effect of the shock waves on the collapses of other bubbles. First, we need to obtain the pressure behind the shock wave. An incompressible model (Supponen et al., 2017) for the pressure distribution around the bubble is used as follows:

$$
\frac{P_{S}}{P_{0}}=1+\frac{R}{3 r}\left(\frac{R_{0}^{3}}{R^{3}}-4\right)-\frac{R^{4}}{3 r^{4}}\left(\frac{R_{0}^{3}}{R^{3}}-4\right)
$$

where $r$ is the radial distance from the bubble center, $P_{0}$ is the atmosphere, $R_{0}$ and $R$ is the bubble radius at $t=0$ and $t=t_{1}$, respectively. In the present study, $r$ is $10.4 \mathrm{~mm}$, the distance between two neighbor bubbles and $R$ is the minimum radius since the strongest shock waves are induced during the bubble collapse. As a result, $P_{\mathrm{s}}$, the pressure of shock wave acting on the inner bubbles is about $4.92 \times 10^{5} \mathrm{~Pa}$. The energy of the shock wave within the area $4 \pi r^{2}$ can be calculated in Eq. (21),

$E_{S}=\frac{\Delta V P_{S}^{2}}{\rho c^{2}}$

where $\Delta V=4 \pi r^{2} \Delta d$ is the volume of the compressed liquid, $r$ is the density and $c$ is the sound speed in water. Hence, the ratio of the energy, $E_{\mathrm{b}}$ acting on a bubble to the whole energy of the shock wave $E_{\mathrm{s}}$, is obtained as follows:

$\frac{E_{b}}{E_{S}}=\frac{4 \pi R_{0}^{2}}{4 \pi r^{2}} \approx 2.5 \times 10^{-4}$

According to the above results, the $E_{\mathrm{b}}$ is enough low comparing with $E_{\mathrm{S}}$ so that we can ignore the action of the shock wave interacting on the inner bubble. As a result, it suggests that the shock pressure induced by the collapse of single bubble is ignored for the collapse of the bubble cluster in the present study. However, it is thought that the shock pressure can play an important role in the collapse of the bubble cluster in other conditions.

The volume variation rate of bubble cluster is recorded during the calculation and then averaged by time. The following discussions are conducted around non-dimensional time-averaged volume variation rate.

The parameters are listed as follows:

Volume faction $\alpha: 0.150,0.268,0.500$

Population of bubbles $N: 3^{3}, 4^{3}, 5^{3}, 6^{3}$

Non-dimensional pressure $p^{\prime}: 0.6,0.7,0.8,0.9$

\subsection{Characteristics of bubble collapse process}

Fig. 5 shows the collapse of the bubble cluster when $\alpha=0.15$, $N=5^{3}$, and $p^{\prime}=0.8$. The initial pressure inside the computational domain is set as $p_{B}$, and the pressure at the outer boundary is $p_{\infty}$. As shown in Fig. 4, the collapse of the bubble cluster starts with the outer regions. The outer bubbles deform and the generated jettings are pointing towards the cluster center. It is found that a high pressure zone around the first layer is induced as shown in green area in Fig. 5(a). However, at this time the centermost bubbles retain a much larger volume, and are still in a relatively lowpressure region (blue area in Fig. 5(a)). This suggests that outer bubbles shield the inner bubbles from collapsing in the beginning and then generate a higher-pressure area to drive the collapses of the inner bubbles. Therefore, the collapse speed of second layer bubbles will increase and result in a higher pressure zone (orange area in Fig. 5(b)). And at last the highest collapse pressure is generated by the center bubble (red area in Fig. 5(b)).

For the case $\alpha=0.15, N=5^{3}$, and $p^{\prime}=0.8$, the nondimensional collapse time of the first layer, second layer, and the center bubble is approximately $2.8,1.2$, and 0.3 , respectively (Fig. 6). The simulation results indicate that bubble cluster is collapsing approximately layer by layer. The collapse of the inner bubbles will be accelerated since a high-pressure zone is generated around them due to the outer bubbles. To understand the behaviors of the bubble collapse in the clusters, we also investigate 


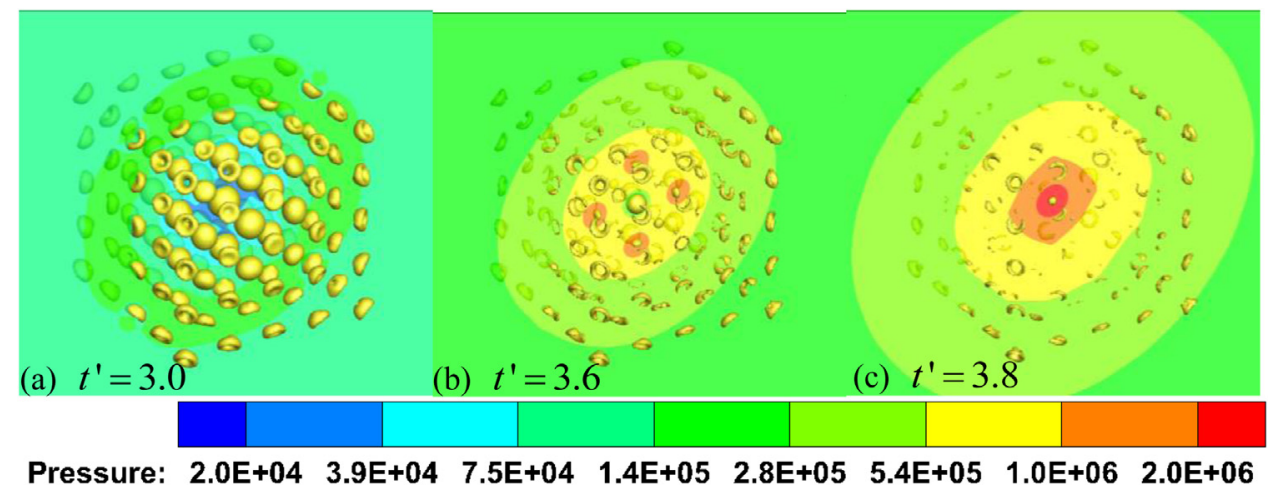

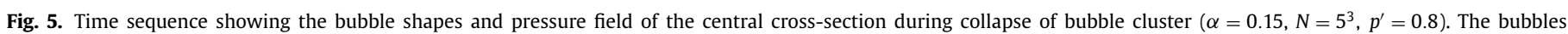
are represented by isosurface of gas fraction with the value of 0.5 , and the pressure contours of the central cross-section range from $2.0 \times 10^{4}$ Pa to $2.0 \times 10^{6}$ Pa.

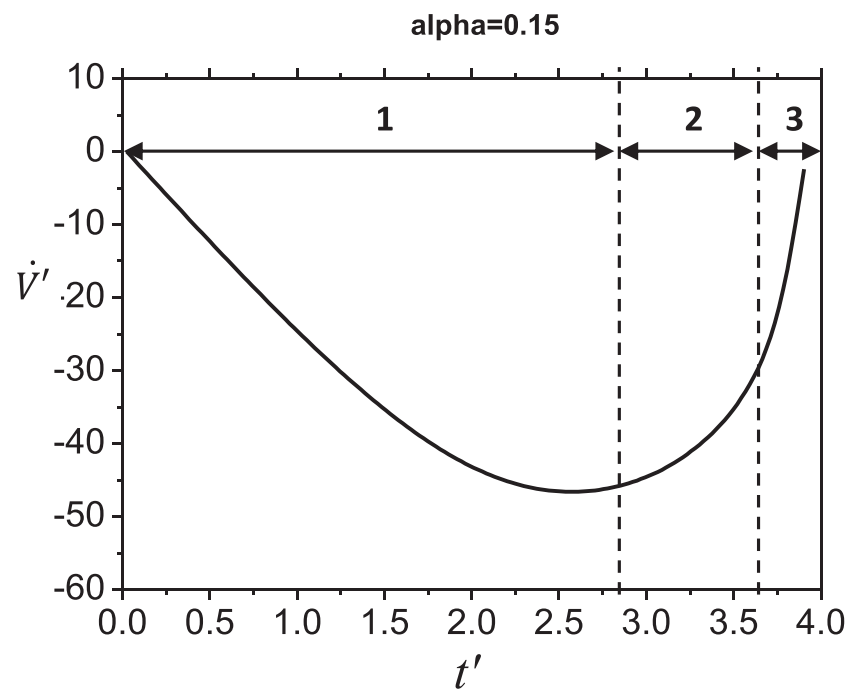

Fig. 6. Non-dimensional collapse speed of bubble cluster $\left(\alpha=0.15, N=5^{3}, p^{\prime}=\right.$ $0.8)$. The non-dimensional collapse speed is defined as $\dot{V}^{\prime}=\dot{V} / \dot{V}_{0}$, where $\dot{V}$ and $\dot{V}_{0}=\frac{4}{3} \pi R_{0}^{3} / T_{C}$ represent volume variation rate of bubble cluster and single isolated bubble respectively. The non-dimensional time is given by $t^{\prime}=t / T_{C}$, where $T_{C}=0.915 R_{0} \sqrt{\frac{\rho_{L}}{p_{\infty}-p_{B}}}$ is the collapse time of an isolated single bubble. The history of collapse speed is divided into three phases: 1 . Collapse of first layer bubbles, 2 . Collapse of second layer bubbles, 3 . Collapse of central bubble.

the influences of bubble population $N$, void fraction $\alpha$ and nondimensional pressure $p^{\prime}$ on collapse speed in the following subsections.

\subsection{Influence of bubble population}

The non-dimensional volume variation rate $\overline{\dot{V}}=\frac{\frac{1}{T} \int_{0}^{T} \dot{V} d t}{\dot{V}_{0}}$ is used to represent the collapse speed of the bubble clusters and measured in terms of the number of the isolated bubbles. Here $T$ is the collapse time of the bubble cluster. For example, $\dot{V}=-10$ means that the volume variation of the bubble cluster is equal to the collapse of 10 isolated bubble at the same time. Table 1-3 present the average collapse speed of the bubble cluster $\bar{V}$ under different bubble populations and void fraction $\alpha$. The void fraction is adjusted by varying the initial bubble radius $R_{B}$ and bubble distance $D_{B}$. Here $D_{B}$ is the distance between the centers of two neighbor bubbles. The power-law exponent $k_{2}=0.68$ is obtained according to the curve fitting, which means the absolute value of the volume variation rate increases with the bubble number. The simulation results show that all the cases considered in the present work have
Table 1

Collapse speed $\overline{\dot{V}}^{\prime}$ for different bubble populations with $\alpha=0.15$.

\begin{tabular}{lllll}
\hline Parameters & & & & \\
\hline$N$ & $3 \times 3 \times 3$ & $4 \times 4 \times 4$ & $5 \times 5 \times 5$ & $6 \times 6 \times 6$ \\
$R_{B}(\mathrm{~mm})$ & 6.87 & 5.15 & 4.12 & 3.43 \\
$D_{B}(\mathrm{~mm})$ & 20.8 & 15.6 & 12.5 & 10.4 \\
$\overline{\dot{V}}^{\prime}$ & -10.7 & -19.6 & -31.4 & -45.6 \\
\hline
\end{tabular}

Table 2

Collapse speed $\overline{\dot{V}}^{\prime}$ for different bubble populations with $\alpha=0.27$.

\begin{tabular}{lllll}
\hline Parameters & \multicolumn{5}{l}{} \\
\hline$N$ & $3 \times 3 \times 3$ & $4 \times 4 \times 4$ & $5 \times 5 \times 5$ & $6 \times 6 \times 6$ \\
$R_{B}(\mathrm{~mm})$ & 8.33 & 6.25 & 50.00 & 4.16 \\
$D_{B}(\mathrm{~mm})$ & 20.8 & 15.6 & 12.5 & 10.4 \\
$\overline{\dot{V}}^{\prime}$ & -9.97 & -18.2 & -29.0 & -42.1 \\
\hline
\end{tabular}

Table 3

Collapse speed $\overline{\dot{V}}^{\prime}$ for different bubble populations with $\alpha=0.5$.

\begin{tabular}{lllll}
\hline Parameters & \multicolumn{5}{l}{} \\
\hline$N$ & $3 \times 3 \times 3$ & $4 \times 4 \times 4$ & $5 \times 5 \times 5$ & $6 \times 6 \times 6$ \\
$R_{B}(\mathrm{~mm})$ & 10.3 & 7.69 & 6.15 & 5.13 \\
$D_{B}(\mathrm{~mm})$ & 20.8 & 15.6 & 12.5 & 10.4 \\
$\overline{\dot{V}}^{\prime}$ & -9.05 & -16.4 & -25.9 & -37.3 \\
\hline
\end{tabular}

a similar trend of the collapse process: the bubble clusters collapse layer by layer. Therefore, combining with the definition of $\overline{\dot{V}}$, the absolute value of $\bar{V}$ increases with the bubble number.

\subsection{Influence of void fraction}

Similarly, we can provide the power-law exponent of $\alpha$ using the data of Table 4.

The curve fitting shows that the power-law exponent of $\alpha$ is $k_{1}=-0.2$, which suggests that the collapse speed decreases with the increase of the void fraction. A high value of $\alpha$ indicates a dense bubble distribution. The inner bubbles reduce the collapse

Table 4

Collapse speed $\overline{\dot{V}}^{\prime}$ for different bubble populations and void fraction $\alpha$.

\begin{tabular}{lllll}
\hline$N$ & $\alpha$ & & & \\
\cline { 2 - 5 } & $3 \times 3 \times 3$ & $4 \times 4 \times 4$ & $5 \times 5 \times 5$ & $6 \times 6 \times 6$ \\
\hline 0.15 & -10.7 & -19.6 & -31.4 & -45.6 \\
0.27 & -9.97 & -18.2 & -9.0 & -42.1 \\
0.5 & -9.05 & -16.4 & -25.9 & -37.3 \\
\hline
\end{tabular}



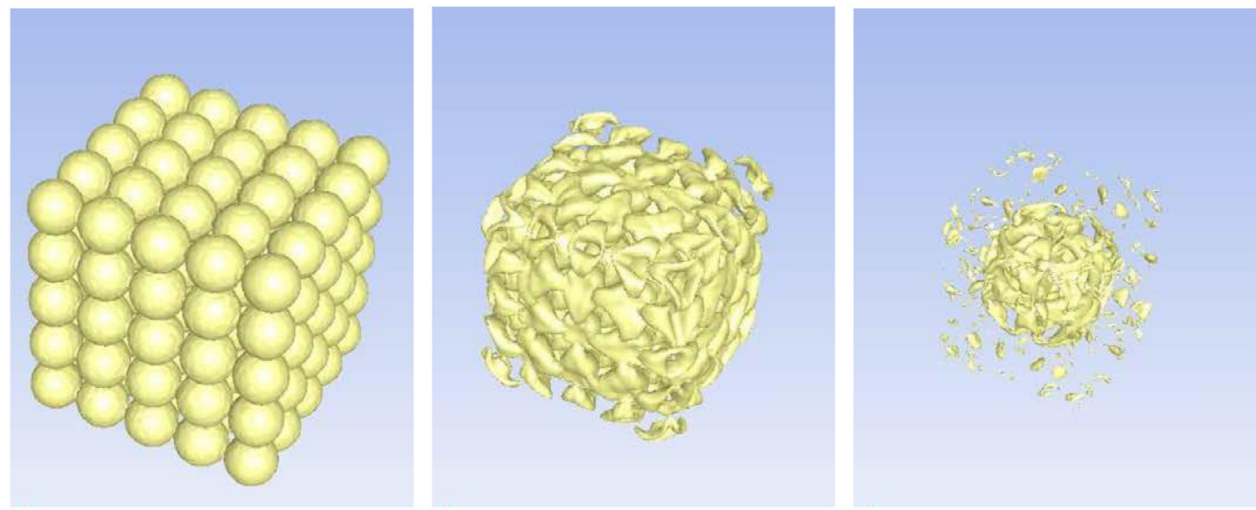

Fig. 7. Collapse of bubble cluster $\left(\alpha=0.5, N=5^{3}\right)$.
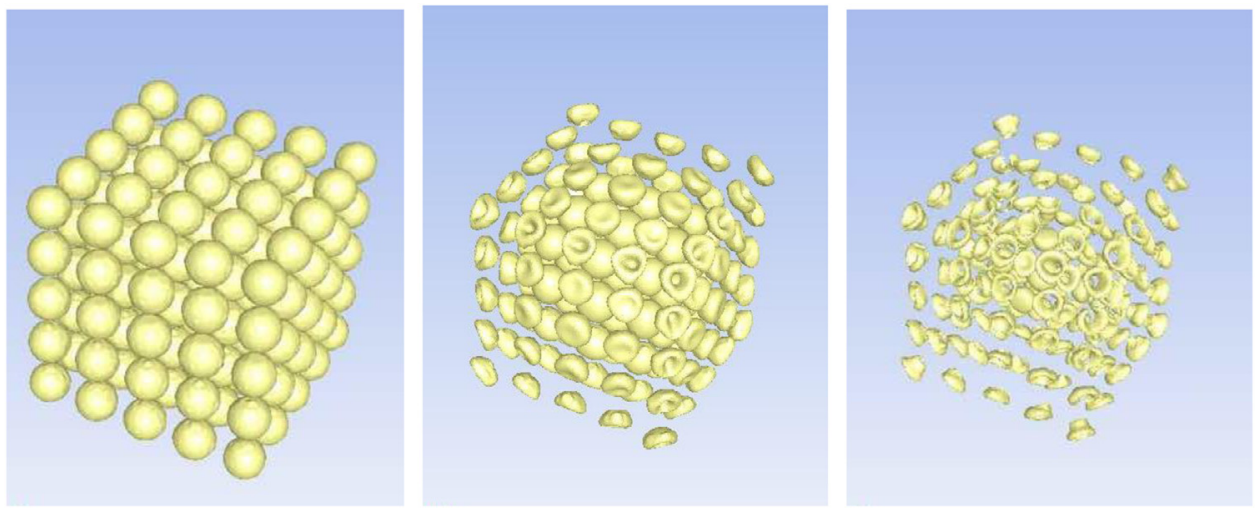

Fig. 8. Collapse of bubble cluster $\left(\alpha=0.27, N=5^{3}\right)$.
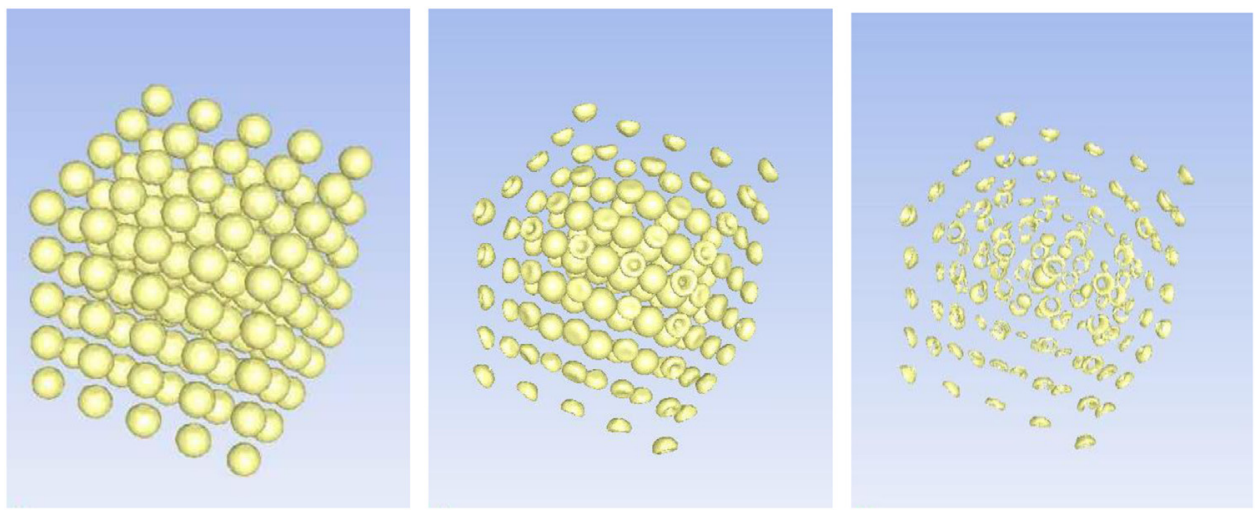

Fig. 9. Collapse of bubble cluster $\left(\alpha=0.15, N=5^{3}\right)$.

velocity of outer bubbles. By contrast, the outer layer of the bubbles shields the inner bubbles from collapsing. Consequently, the collapsing speed slows down with the rise of the void fraction. By comparing the collapse process under the conditions of different void fractions, it is found that the bubble cluster trends to collapse layer by layer with a high void fraction (Fig. 7- Fig. 9). And bubble coalescence occurs in the case of $\alpha=0.5, N=5^{3}$ (Fig. 7).

\subsection{Influence of non-dimensional pressure}

The power-law exponent of $p^{\prime}=0.61$ is obtained according to the curve fitting, which indicates that the collapse increases with the non-dimensional pressure (Table 5). Non-dimensional pressure represents the driving force for the collapse of the bubble cluster. As shown in Fig. 11, the central bubble begins to shrink when
Table 5

Collapse speed $\overline{\bar{V}}^{\prime}$ for different pressures with $\alpha=0.27$.

\begin{tabular}{lllll}
\hline Parameters & \multicolumn{5}{l}{} \\
\hline$\alpha$ & 0.27 & 0.27 & 0.27 & 0.27 \\
$N$ & $3 \times 3 \times 3$ & $3 \times 3 \times 3$ & $3 \times 3 \times 3$ & $3 \times 3 \times 3$ \\
$p^{\prime}$ & 0.6 & 0.7 & 0.8 & 0.9 \\
$\overline{\dot{V}}^{\prime}$ & -8.31 & -9.12 & -9.97 & -10.7 \\
\hline
\end{tabular}

the outer bubbles are collapsing. By comparing with the process of the bubble collapse in Fig. 10, the collapse become violent with the increase in $p^{\prime}$. One of the reasons can be related closely to the pressure difference between outside and inside the central bubble. Furthermore, it is found that a higher $p^{\prime}$ leads to faster collapse of the bubble cluster. 

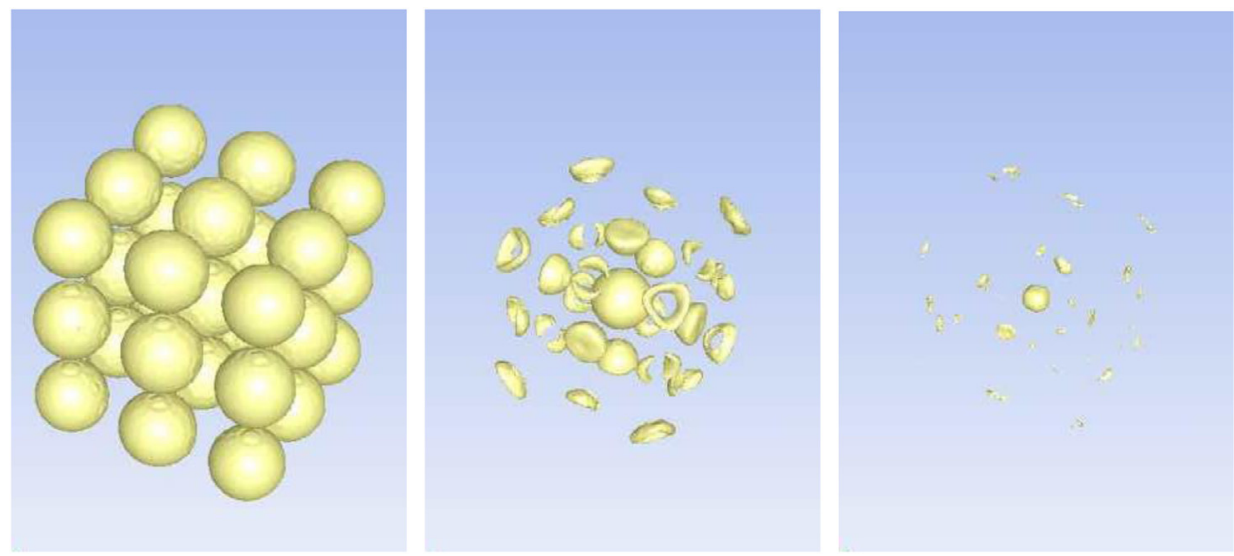

Fig. 10. Collapse of bubble cluster $) p^{\prime}=0.9($.
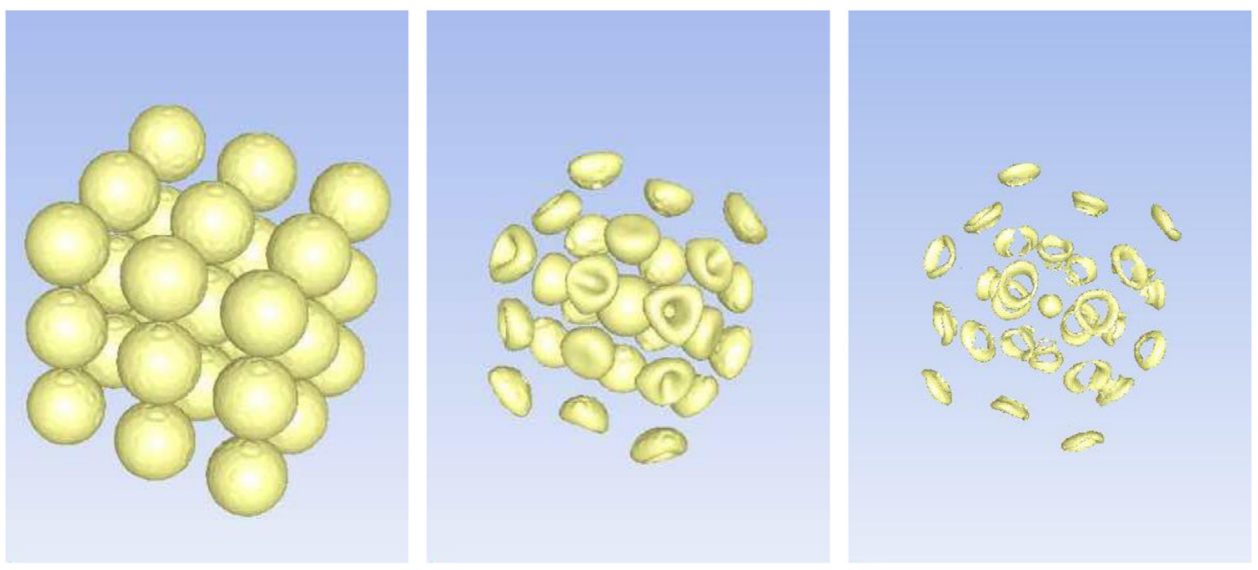

Fig. 11. Collapse of bubble cluster $\left(p^{\prime}=0.8\right)$.

\subsection{Empirical formula for collapse speed of bubble cluster}

Based on the theoretical analysis and simulation results, the constant coefficient $c$ is close to 1 . Combining with the influence of $\alpha, N$, and $p^{\prime}$, Eq. (3) becomes:

$$
\overline{\dot{V}^{\prime}}=\alpha^{-0.2} N^{0.68} p^{0.61}
$$

Eq. (23) gives the non-dimensional collapse velocity of the bubble cluster. We can also derive a condensation rate for the cavitation model. First, the change rate of the void fraction can be provided as:

$\dot{\alpha}=\frac{\overline{\dot{V}}}{V_{m}}=-0.0039 \alpha^{-0.2} N^{0.68} p^{0.61} \frac{\frac{4}{3} \pi R_{0}^{3}}{V_{m} T_{C}}$

After arrangement, we can obtain the condensation rate as follows:

$\dot{m}^{-}=-C_{c} \frac{\rho_{l} \rho_{v}}{\rho_{m}}\left(\frac{n^{0.013}}{V_{m}^{0.32} \alpha^{0.53}}\right) \alpha\left(\frac{p-p_{v}}{p}\right)^{0.61} \sqrt{\frac{2\left(p-p_{v}\right)}{3 \rho_{l}}}$,

where $V_{m}$ is the total volume, including bubbles and surrounding liquid, and $C_{C}=0.057$.

\section{Influence of distributions}

Primitive cubic lattice is the thinnest regular distribution. In this part, different arrangements of the bubbles are used to analyze the representativeness of the primitive cubic lattice distribution, including the random distribution and right square pyramid
Table 6

Collapse speed $\overline{\dot{V}}^{\prime}$ and max pressure $P_{\max } / p_{0}$ for initial different arrangements.

\begin{tabular}{lllll}
\hline Case & Distribution & $\overline{d^{\prime}}$ & $\overline{\dot{V}}$ & $P_{\max } / p_{0}$ \\
\hline 1 & Cubic lattice & 3.45 & 29.0 & 40.5 \\
2 & Random & 3.44 & 28.8 & 30.9 \\
3 & Random & 3.40 & 28.7 & 28.2 \\
4 & Random & 3.36 & 28.6 & 27.6 \\
5 & Random & 3.30 & 28.3 & 25.7 \\
6 & Random & 3.24 & 27.8 & 24.7 \\
7 & Random & 3.20 & 27.5 & 29.8 \\
8 & Square pyramid & 3.12 & 27.6 & 19.7 \\
\hline
\end{tabular}

lattice distribution. As shown in Fig. 12, all the sides are equilateral triangles in the case of the right square pyramid.

To distinguish different random distributions, a dimensionless bubble distance is defined as:

$\bar{d}^{\prime}=\frac{\bar{d}}{2 R_{B}}$

where $\frac{1}{d}=\sum_{k=1}^{n} \frac{1}{\bar{d}_{i}}$ and $\frac{1}{\bar{d}_{i}}=\sum_{k=1}^{n, k \neq i} \frac{1}{d_{i k}}$.

The bubble-bubble interaction strengthens as their distance decreases. Thus, harmonic average is adopted to define the average distance. The collapse speed $\ddot{\dot{V}}$ and max pressure $P_{\max } / p_{0}$ for different arrangements (bubble size and average bubble distance) are listed in Table 6 . The corresponding distributions of bubble clusters are shown in Fig. 13. 


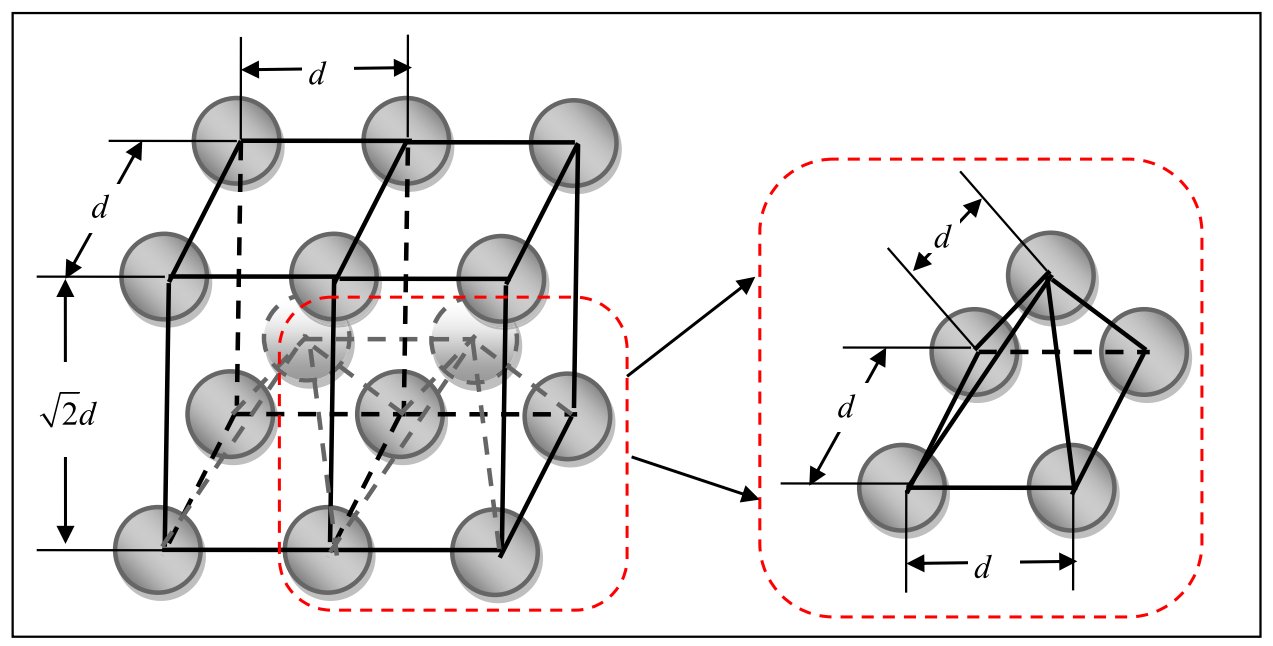

Fig. 12. Square pyramid lattice.
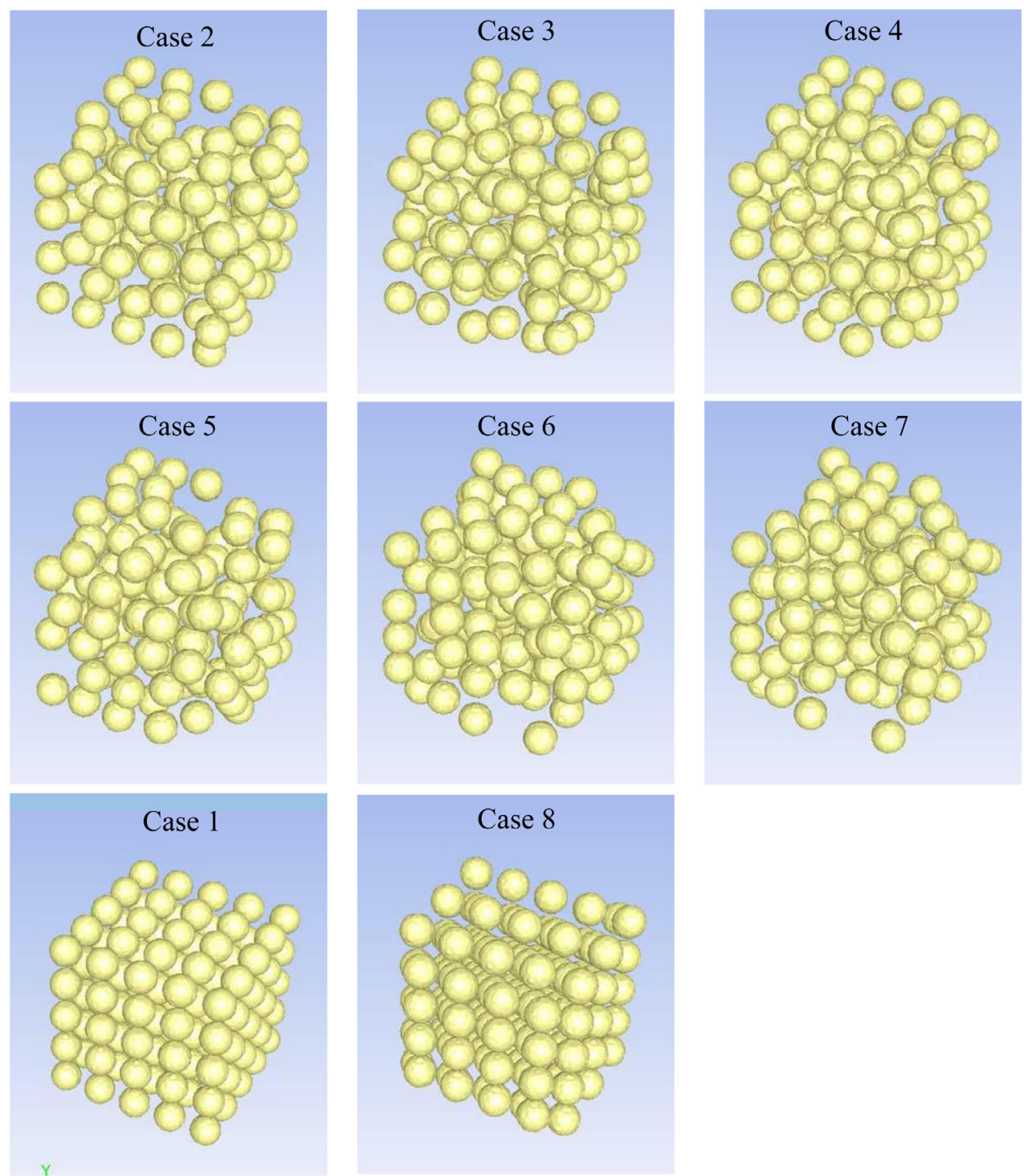

Fig. 13. Initial distributions of bubble clusters. 


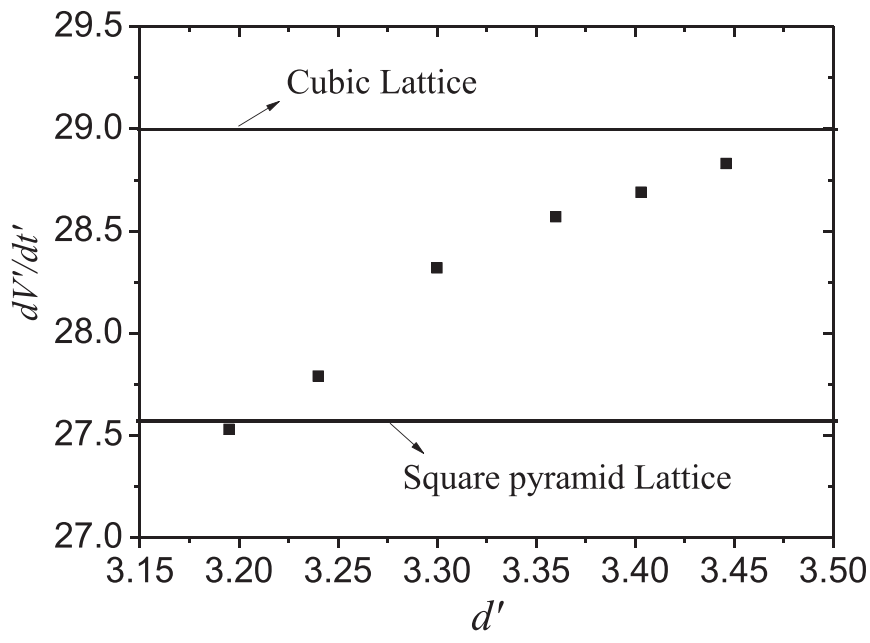

Fig. 14. Collapse velocity of bubble cluster.

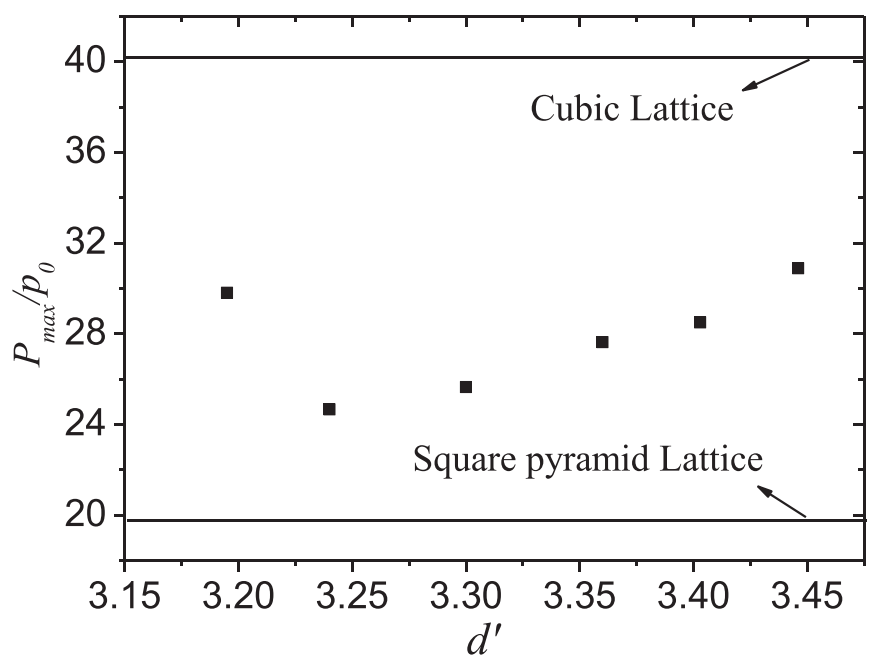

Fig. 15. Maximum pressure during collapse.

From the Fig. 14, we can see that the collapse velocity slows down as the $\bar{d}^{\prime}$ increases since the outer bubbles shield the inner bubbles from collapsing. The collapse velocities and maximal pressure for the random distributions are located between the primitive cube distribution and right square pyramid distribution, as shown in Fig. 14 and Fig. 15. On the other hand, it is found that the maximum pressure does not increase monotonously with the rising of the dimensionless bubble distance. This finding may be related to the local distribution of the bubbles, which needs further study and is not discussed in this study. For the cases considered in the present work, the relative error of the collapse velocity for the primitive cube distribution and right square pyramid distribution is $5.2 \%$, and the collapse velocity of the random distributions are between them. This result indicates that the primitive cube distribution gets closest to the actual distribution of the bubble cluster, and the condensation rate constructed in the present paper can be used in simulation of cavitating flow. The performance of this condensation rate will be conducted in the following study.

\section{Conclusions}

We focus on the bubble-bubble interaction in the collapse stage of cavitation cloud. Non-dimensional parameters for collapse of bubble cluster are acquired by dimensional analysis, including bubble quantity, volume of fraction, and dimensionless pressure. Prim- itive cubic lattice distribution is selected to examine the influence of these parameters. An empirical formula for collapse speed of bubble cluster is achieved by direct numerical simulation based on the VOF and the LES. The results imply the following:

1) Bubble cluster will collapse approximately layer by layer.

2) Large $n$ will result in a large volume variation rate of bubble cluster.

3) The collapsing speed of bubble cluster will slow down with the increase in volume fraction.

The collapse of bubble clusters with right square and random distributions are simulated to discuss the representativeness of primitive cube distribution and the applicability of the proposed empirical formula. Results show that the collapse speed and maximal pressure for random distributions are located between that for primitive cube distribution and right square pyramid distribution. The relative error of collapse speed for primitive cube distribution and right square pyramid distribution is less than $5 \%$. This finding indicates that bubble cluster with primitive cube distribution has certain representativeness of actual bubble cluster. The condensation rate set in this study can be used in the simulation of cavitating flow in the fields of high-speed vehicles, ultrasonic treatments, and shock waves lithotripsy.

\section{Declaration of Competing Interest}

The authors declare that they have no known competing financial interests or personal relationships that could have appeared to influence the work reported in this paper.

\section{CRediT authorship contribution statement}

Tezhuan Du: Conceptualization, Data curation, Visualization, Writing - original draft. Jingzhu Wang: Validation, Writing - review \& editing. Yiwei Wang: Methodology. Chenguang Huang: Supervision.

\section{Acknowledgments}

The authors acknowledge the financial support from the National Natural Science Foundation of China (11872065, 11402276).

\section{Supplementary materials}

Supplementary material associated with this article can be found, in the online version, at doi:10.1016/j.ijmultiphaseflow.2020. 103322.

\section{References}

ANSYS Fluent12, 2008, 1 User's Guide. U.S. ANSYS Inc.

Bark, G., Grekula, M., Bensow, R., et al., 2011. On Some Physics to Consider in Numerical Simulation of Erosive Cavitation. International Symposium on Cavitation, Singapore.

Bensow, R.E., Bark, G.R., 2010. Implicit LES predictions of the cavitating flow on a propeller. ASME J. Fluids Eng. 132, 041302.

Bensow, R.E., Bark, G., 2010. Simulating cavitating flows with LES in OpenFOAM. V European Conference on Computational Fluid Dynamics.

Bremond, N., Arora, M., Ohl, C.D., Lohse, D., 2006. Controlled multibubble surface cavitation. Phys. Rev. Lett. 96 (22), 224501.

Chahine, G.L., Hsiao, C.-.T., Raju, R., 2014. Scaling of Cavitation Bubble Cloud Dynamics on Propellers. In: Kim, K.-H., Chahine, G.L., Franc, J.-P., Karimi, A. (Eds.), Advanced Experimental and Numerical Techniques for Cavitation Erosion Prediction, Chapter 15. Springer, Berlin, pp. 345-373.

Chahine G, L., Duraiswami, R., 1992. Dynamical interactions in a multi-bubble cloud. J. Fluids Eng. 114 (4), 680.

Coutier-Delgosha, O., Stutz, B., Vabre, A., Legoupil, S., 2007. Analysis of cavitating flow structure by experimental and numerical investigations. J. Fluid. Mech. 578, $171-222$. 
D'Agostino, L., Brennen, C.E., 1989. Linearized dynamics of spherical bubble clouds. J. Fluids Mech. 199, 155-176.

Doinikov, A.A., 2004. Mathematical model for collective bubble dynamics in strong ultrasound fields. J. Acoust. Soc. Am. 116 (2), 821.

Hsiao, C.-T., Jingsen, Ma, Chahine, G.L., May 2018. A parametric study of bubble cloud dynamics near a wall in an acoustic field. Proceeding of International Symposium on Cavitation.

Huang, B., Ducoin, A., Yin L, Y., 2012. Evaluation of cavitation models for prediction of transient cavitating flows around a stationary and a pitching hydrofoil. In: 8th International Symposium on Cavitation, pp. 601-608.

Huang, B., Ducoin, A., Yin L, Y., 2013. Physical and numerical investigation of cavitating flows around a pitching hydrofoil. Phys. Fluids 25 (10), 2109.

Huang, B., Zhao, Y., Wang, G., 2014. Large Eddy Simulation of turbulent vortex-cavitation interactions in transient sheet/cloud cavitating flows. Comput. Fluids 92 (3), 113-124.

Ji, B., Luo, X., Peng, X., et al., 2012. Numerical analysis of cavitation evolution and excited pressure fluctuation around a propeller in non-uniform wake. Int. J. Multiph. Flow 43 (43), 13-21.

Kedrinskii, H.,.V., Mørch, K., 1725. On the dynamics of cavity clusters. J. Phys. D. Appl. Phys. 15 (9), 1982.

Kim, K.H., Chahine, G., Franc, J.P., Karimi, A., 2014. Advanced experimental and numerical techniques for cavitation erosion prediction. Fluid Mechanics and Its Applications 106.

Kubota, A., Kato, H., 1989. Unsteady structure measurement of cloud cavitation on a foil section. J. Fluids Eng. 111 (3), 204-210.

Kunz, R.F., Boger, D.A., Stinebring, D.R., Chyczewski, T.S., Lindau, J.W., Gibeling, H.J., Venkateswaran, S., Govindan, T.R., 2000. A preconditioned Navier-Stokes method for two-phase flows with application to cavitation prediction. Comput. Fluids 29 (8), 849-875.

Ma, J., Hsiao, C.-.T., Chahine, G.L., January 2018. Numerical study of acoustically driven bubble cloud dynamics near a rigid wall. Ultrason. Sonochem. 40, 944-954.

Ma, J., Chahine, G.L., Hsiao, C.-.T., May 2015. Spherical bubble dynamics in a bubbly medium using an Euler-Lagrange model. Chem. Eng. Sci. 128, 64-81 Vol..

Maeda, K., Colonius, T., 2018. Eulerian-Lagrangian method for simulation of cloud cavitation. J. Comput. Phys. 37, 904-1017.

Maiga, M.A., Coutier-Delgosha, O., Buisine, D., 2018. A new cavitation model based on bubble-bubble interactions. Phys. Fluids, 123301.

Merkle, C.L., Feng, J., Buelow, P.E.O., 2001. Computational modeling of the dynamics of sheet cavitation. In: Proceedings of 3rd International Symposium on Cavitation. Grenoble, France.
Morgut, M., Nobile, E., Biluš, I., 2011. Comparison of mass transfer models for the numerical prediction of sheet cavitation around a hydrofoil. Int. J. Multiph. Flow 37 (6), 620-626.

Omta, R., 1987. Oscillations of a cloud of bubbles of small and not so small amplitude. J. Acoust. Soc. Am. 82, 1018-1033.

Owis F, M., Nayfeh, A.H., 2004. Numerical simulation of 3-D incompressible, multi-phase flows over cavitating projectiles. Eur. J. Mech. B Fluids 23 (2), 339-351.

Schnerr, G.H., Sauer, J., 2001. Physical and numerical modeling of unsteady cavitation dynamics. 4th International Conference on Multiphase Flow.

Singhal, A.K., Athavale, M.M., Li, H., Jiang, Y., 2002. Mathematical basis and validation of the full cavitation model. J. Fluids Eng. 124 (3), 617-624.

Supponen, O., Obreschkow, D., Kobel, P., Tinguely, M., Dorsaz, N., Farhat, M., 2017. Shock waves from non-spherical cavitation bubbles. Phys. Rev. Fluids 2, 093601

Tiwari, A., Freund, J.B., Pantano, C., 2013. A diffuse interface model with immiscibility preservation. J. Comput. Phys. 252, 290-309.

Tiwari, A., Pantano, C., Freund J, B., 2015. Growth-and-collapse dynamics of small bubble clusters near a wall. J. Fluid. Mech. 775, 1-23.

van Wijngaarden, L., 1964. On the collective collapse of a large number of cavitation bubbles in water. 11th Int. Congr. of Appl. Mech.

Wang, G., Ostoja-Starzewski, M., 2007. Large Eddy simulation of a sheet/cloud cavitation on a NACA0015 hydrofoil. Appl. Math. Model. 31 (3), 417-447 2007.

Xu, C., Wang, Y.W., Huang, C.G., Yu, C., Huang, J., 2018. Analysis of near-wall effect on cloud cavitating flow that surrounds an axisymmetric projectile using large eddy simulation with Cartesian cut-cell mesh method. Eur. J. Mech. B Fluids 67, $15-24$.

Yakubov, S., Maquil, T., Rung, T., 2015. Experience using pressure-based CFD methods for Euler-Euler simulations of cavitating flows. Comput. Fluids 111, 91-104.

Yasui, K., Kato, K., 2012. Bubble dynamics and sonoluminescence from helium or xenon in mercury and water. Phys. Rev. E 86, 036320.

Zhang, A., Yao, X., Li, J., 2008. The dynamics of bubbles. Acta Physica Sinica 57 (3), $1672-1682$.

Zhang, L., Wen, Z., Shao, X., 2013. Investigation of bubble-bubble interaction effect during the collapse of multi-bubble system. Chin. J. Theor. Appl. Mech. 45 (6), 861-867.

Zwart, P.J., Gerber, A.G., Belamri, T., 2004. A two-phase flow model for predicting cavitation dynamics. 7th International Conference on Multiphase Flow. Yokohama, Japan. 